\title{
AiMT
}

Advances in Military Technology

Vol. 12, No. 2 (2017), pp. 281-300

ISSN 1802-2308, eISSN 2533-4123

DOI 10.3849/aimt.01207

\section{Robust Autopilot Design and Hardware-in-the-Loop Simulation for Air to Air Guided Missile}

\author{
A. Mohamed ${ }^{1 *}$, G.A. El-Sheikh ${ }^{2}$, A.N. Ouda ${ }^{3}$ and A.M. Youssef ${ }^{3}$ \\ ${ }^{1}$ University of Ontario Institute of Technology, Oshawa, Canada \\ ${ }^{2}$ Science Valley Academy, Cairo, Egypt \\ ${ }^{3}$ Military Technical College, Cairo, Egypt
}

\begin{abstract}
The manuscript was received on 19 July 2017 and was accepted after revision for publication on 24 November 2017.
\end{abstract}

\begin{abstract}
:
This paper proposed a robust autopilot design for air to air guided missile and a Hardware-in-the-Loop (HIL) simulation which is based on the derived missile-control transfer functions and the 6DOF simulation model. The introduced autopilot is implemented within the 6DOF simulation to check its robustness against non-modeled dynamics and nonlinearities. The nonlinear 6DOF equations of motions are solved together to obtain the pitch and yaw transfer functions. The missile equations are described in the form of modules programmed within the $C++$ environments to form the baseline for subsequent design and analysis. Furthermore, a comparison between both our previous work, i.e. classical and robust autopilot, are justified via HIL simulation. The simulation results demonstrated the robustness capability in presence disturbance and noise.
\end{abstract}

\section{Keywords:}

homing guidance systems, modelling and simulation, autopilot design, robust control, HIL simulation

\section{Notation}

6DOF Six Degrees of Freedom

LFT Linear Fractional Transformation

$\left|W_{p}^{-1}(j \omega)\right| \quad$ Desired disturbance attenuation factor

$\left|W_{u}(j \omega)\right| \quad$ Size of the largest anticipated additive plant perturbation

$\left|W_{t}(j \omega)\right| \quad$ Size of the largest anticipated multiplicative plant perturbation

$\left\|T_{y 1 u 1}\right\|_{\infty} \quad$ Mixed-sensitivity cost function

\footnotetext{
* Corresponding author: Faculty of Engineering and Applied Science, University of Ontario Institute of Technology (UOIT), Canada.E-mail: Amr.Mohamed@uoit.net
} 


\section{Introduction}

The great developments in mathematics and computational capabilities facilitate the design and implementation of control. In addition, the huge developments in nanotechnology and its availability in civilian applications with less cost, size and weight attract many of researchers all over the world towards embedded systems, especially the embedded flight control. Among the real applications are the guided missiles, particularly the air-to-air guided missile systems which are self-commanded towards air targets.

Future homing missiles require to cope with demands for greater range and higher manoeuvrability which can be achieved by an increase in the robustness and the performance of missile autopilot. Various design techniques are used to enhance the missile autopilot performance. On the other hand, the recent advances in robust control theory [1-3] have the capability to achieve a robust performance and stability which are essential for the design of next generation of missiles autopilots. There are different benefits from using robust control design approach, such as a better flexibility in the choice of airframe geometry and more tolerance to uncertainty in the missile autopilot design.

Several researches for missile autopilot design have been reviewed, e.g. Li introduced a straightforward decoupling control method based on robust state feedback control algorithm and disturbance observer developed for the bank to turn missile autopilot design [4]. The bank to turn missile dynamic systems was divided into three sub-systems in terms of roll, yaw, and pitch. Then disturbance observer was developed for each sub-system in order to estimate both the nonlinear couplings and the external disturbances. The robust state feedback control laws were introduced to stabilize linear parts of each sub-system. Mattei [5] developed a robust nonlinear controller for a highly manoeuvrable missile. The introduced robust back-stepping controller was applied to the MIMO model in order to achieve Both Bank to Turn (BTT) and Skid to Turn (STT) manoeuvres. Liu [6] investigated the problem related to composite antidisturbance autopilot design for missile systems with multiple disturbances. For this purpose, the nonlinear disturbance is observed to estimate the disturbances. The finite time integral sliding mode control method was introduced to develop a feedback controller. Lee and Singh developed a nonlinear adaptive longitudinal autopilot to control the angle of attack of the missile in the presence of uncertainties and external disturbance [7].

The design and analysis of an autopilot to satisfy the ever-increasing performance requirements necessitates the availability of the guided missile system model that consists of the different equations representing its spatial motion in space. These equations consist of nonlinear differential equations in addition to geometrical relations and the guidance law. The input stimuli to the model are launch conditions, target motion, and the appropriate guidance law, while the outputs are the missile flight data (velocity, acceleration, range, range rate, turning rate, etc.) during simulated scenario.

The main objective of this paper is to utilize the 6DOF and transfer functions obtained from previous work [8] for autopilot design using robust control and to evaluate its performance against different sources of uncertainty complemented with the HIL evaluation. Towards this objective, Section 2 tailors the $H_{\infty}$ robust control to the intended missile-system for autopilot design. The obtained autopilot is evaluated against different sources of uncertainty including non-modelled dynamics, disturb- 
ances, measurement noise and nonlinearities. The nonlinearities are considered through flight path evaluation presented in Section3. Then, the final design is evaluated with HIL in Section 4. Finally, the conclusions have been drawn in Section 5.

\section{Robust Autopilot Design and Analysis}

The generalized plant $\boldsymbol{P}$, which is defined from the inputs $\left[\begin{array}{ll}u_{1} & u_{2}\end{array}\right]^{\mathrm{T}}$ to the outputs $\left[\begin{array}{ll}y_{1} & y_{2}\end{array}\right]^{\mathrm{T}}$, can be expressed in terms of its state space realization as follows:

$$
\boldsymbol{P}(s)=\left[\begin{array}{ccc}
A & B_{1} & B_{2} \\
C_{1} & D_{11} & D_{12} \\
C_{2} & D_{21} & D_{22}
\end{array}\right] .
$$

It is required to find such a stabilizing feedback control law $\left(u_{2}\right)$ that the norm of the closed-loop transfer function matrix $\left(T_{y 1 u 1}\right)$ is small $[9,10]$, where

$$
\begin{gathered}
u_{2}(s)=C(s) y_{2}(s), \\
T_{y_{1} u_{1}}=P_{11}(s)+P_{12}(s)\left[I-C(s) P_{22}(s)\right]^{-1} C(s) P_{21}(s) .
\end{gathered}
$$

The state-space model is considered so that the closed-loop transfer function matrix is the weighted mixed sensitivity as shown in Fig. 1.

$$
T_{y 1 u 1}=\left[\begin{array}{cc}
W_{p} & S \\
W_{u} & R \\
W_{t} & T
\end{array}\right] .
$$

Where $S, R$ and $T$ are given by [11].

$$
\begin{aligned}
& S=(I+G C)^{-1} \\
& R=C(I+G C)^{-1} \\
& T=G C(I+G C)^{-1}
\end{aligned}
$$

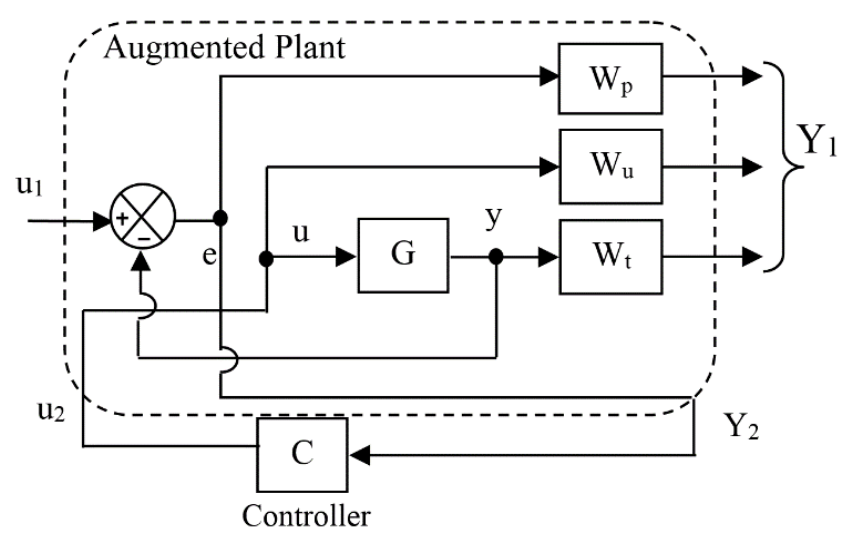

Fig. 1 Augmented Plant $\boldsymbol{P}(s)$

In practice, it is usually not necessary to obtain a true optimal controller, but it is often simpler to find a sub-optimal controller. Let us suppose that $\gamma_{\min }$ is the minimum 
value of $\left\|F_{\ell}(P, C)\right\|_{\infty}$ over all possible stabilizing controllers $C$. Then, the $H_{\infty}$ suboptimal control problem is to find all stabilizing controllers so that

$$
\left\|F_{\ell}(P, C)\right\|_{\infty}<\gamma \text {. }
$$

Where $F_{\ell}(P, C)$ is the lower Linear Fractional Transformation (LFT) of $P$ and $C$ given that $\gamma>\gamma_{\min }$ this problem can be solved efficiently using the algorithm of [12], by reducing $\gamma$ iteratively to yield the optimal solution [13].

\subsection{Weight Selection Methodology}

The methodology of $W_{p}$ and $W_{u}$ calculation is considered in this section. The calculation is based on the compromising between the sensitivity and complementary sensitivity as follows:

A feedback-control system must satisfy certain performance specifications and it must tolerate model uncertainties. The feedback control system has three components: the plant, sensors to measure the plant outputs, and a controller to generate the plant's input or control signal as shown in Fig. 2. Generally, this system has three inputs and three outputs as described in Eq. (6).

In case of unity feedback system, the sensitivity function $(S)$, the complementary sensitivity function $(T)$ and the control sensitivity function $(R)$ are defined as follows:

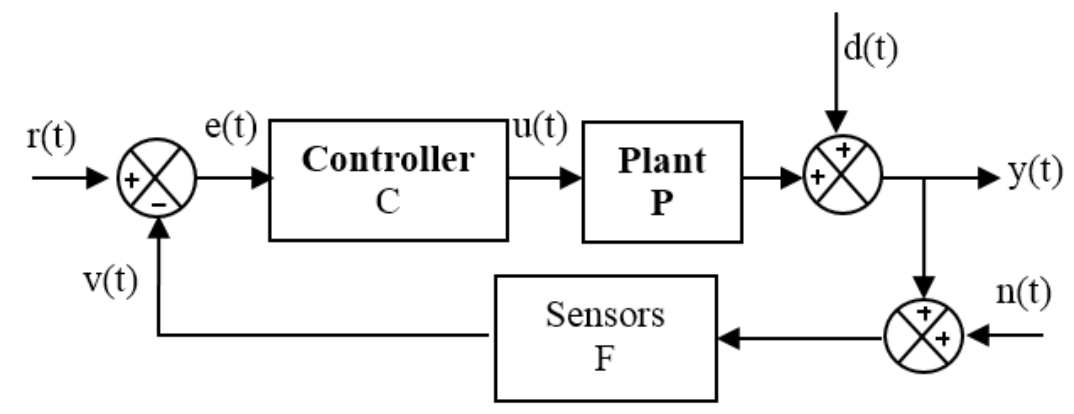

Fig. 2 Feedback Control

$$
S=\frac{1}{1+L}, T=\frac{L}{1+L}, R=\frac{C}{1+L},
$$

where $L$ denotes the loop transfer function $(L=P C)$ and $(T=1-S)$.

The contribution of system inputs to the actual output $y(t)$, the tracking error $e(t)$, and the controller/actuator signal, $u(t)$, are described by the following matrix equation:

$$
\left[\begin{array}{l}
y \\
u \\
e
\end{array}\right]=\left[\begin{array}{ccc}
T & S & -T \\
R & -R & -R \\
S & -S & -S
\end{array}\right]\left[\begin{array}{l}
r \\
d \\
n
\end{array}\right] .
$$

In view of performance requirements and the above relationships, the following conclusions can be drawn:

- Disturbance rejection: The loop gain $(P C)$ should be large to yield small $S$ and to minimize the effects of disturbance. 
- Tracking: The loop gain $(P C)$ should be large to yield small $S$ and to keep tracking errors small.

- Noise suppression: the loop gain should be small to yield small $T$ and consequently to minimize the effects of noise on the system output and tracking errors.

- Actuator limits: $R$ must be bounded to ensure that the actuating signal driving the plant does not exceed plant tolerances. In addition, the control energy should be minimal so that smaller actuators can be used.

Tracking and disturbance rejection requires small sensitivity, but noise suppression requires small complementary sensitivity. However, reducing both transfer functions to zero simultaneously is not possible because these two transfer functions add up to unity $(S+T=I)$. This conflict can be avoided by noticing that, in practice, command inputs and disturbances are low-frequency signals whereas the measurement noise is high-frequency signal. Therefore, both objectives can be met by keeping $S$ small in the low-frequency range and $T$ small in the high frequencies. In addition, the control-energy constraint requires keeping $R$ small, which can be achieved by keeping $T$ small as $R=C S=T / P$.

The general feature of this loop gain is that it has high gain at low frequencies (for good tracking and disturbance rejection) and low gain at high frequencies (for noise suppression).

\subsection{Model Order Reduction for Robust Control}

In control theory, eigenvalues define the system stability, whereas Hankel singular values define the energy of each state in the system. Keeping larger energy states of a system preserves most of its characteristics in terms of stability, frequency, and time responses. Model reduction techniques presented in this paper are based on the Hankel singular values of a system. They can achieve a reduced-order model that preserves the majority of the system characteristics. Mathematically, for given a stable statespace system (A, B, C, D) the Hankel singular values are defined as [14]:

$$
\sigma_{H}=\sqrt{\lambda_{i}}\left(P_{g} Q_{g}\right)
$$

where $P_{g}$ and $Q_{g}$ are controllability and observability satisfying the following equations:

$$
\begin{aligned}
& A P+P A^{\mathrm{T}}=-B B^{\mathrm{T}} \\
& A^{\mathrm{T}} Q+Q A=-C^{\mathrm{T}} C
\end{aligned}
$$

Robust control offers several algorithms for model approximation and order reduction which are used to control the absolute or relative approximation error based on the Hankel singular values of the system. Model reduction approaches can be put into two categories:

- Additive error method in which the reduced-order model $G_{\text {red }}$ has an additive error bounded by an error criterion $\left\|G-G_{\text {red }}\right\|_{\infty}$.

- Multiplicative error method where the reduced-order model has a multiplicative or relative error bounded by an error criterion $\left\|G^{-1}\left(G^{-} G_{\text {red }}\right)\right\|_{\infty}$.

The error is measured in terms of peak gain across frequency $\left(H_{\infty}\right.$ norm), where the error bounds are functions of the neglected Hankel singular values. 


\section{a. Additive Model Reduction}

Three methods are available to do the additive error model reduction [15]:

- Square-root balanced model truncation

- Schur balanced model truncation

- Hankel minimum degree approximation

Each of the above methods possess the same infinity-norm error bound for a $\mathrm{k}^{\text {th }}$ order reduced order model $\widetilde{G}(S)$ of an $\mathrm{m}^{\underline{\underline{t h}}}$ order system $G(s)$ :

$$
\bar{\sigma}\{G(j \omega)-\widetilde{G}(j \omega)\} \leq 2 \sum_{i=k+1}^{m} \sigma_{i} \forall \omega .
$$

\section{b. Multiplicative Model Reduction}

Combining the Balanced Stochastic Truncation (BST) with the Relative Error bound (REM) can result in the optimal solution for robust model reduction [15]. Implements the Schur version of the BST-REM theory and yields the following "relative-error" and "multiplicative-error" bounds [9]:

$$
\begin{aligned}
\left\|G^{-1}(G-\widetilde{G})\right\|_{\infty} & \leq \Delta_{\text {err }} \\
\left\|\widetilde{G}^{-1}(G-\widetilde{G})\right\|_{\infty} & \leq \Delta_{\text {err }} \\
\Delta_{\text {err }} & =\sum_{i=k+1}^{n} \frac{2 \sigma_{i}}{1-\sigma_{i}}
\end{aligned}
$$

One method is available to do the multiplicative error model reduction known by balanced stochastic truncation. In this method, given a state space (A, B, C, D) of a system and the desired reduced order $(k)$, the reduced order state space model steps can be found in more details in [15].

\subsection{Robust Controller Design}

\section{a. First Robust Controller}

A robust controller is designed using the following weights:

$$
W_{p}=\frac{0.6234 s+20}{s+2.0}, W_{t}=\frac{0.03 s+1}{10 s+1.91}, W_{u}=0.00072,
$$

The obtained controller has the form:

$$
C 1=\frac{-1.002 \mathrm{e}^{5} s^{4}-2.921 \mathrm{e}^{7} s^{3}-1.725 \mathrm{e}^{9} s^{2}-7.171 \mathrm{e}^{9} s^{1}-1.307 \mathrm{e}^{9}}{s^{5}+685.7 s^{4}+685.7 s^{3}+6.564 \mathrm{e}^{5} s^{2}+5.587 \mathrm{e}^{5} s+1.933 \mathrm{e}^{4}} .
$$

The frequency response of the obtained weighted sensitivities is shown in Figure 3a, and the closed loop response is shown in Fig. 3b. Due to high order, the model order reduction techniques [16] are applied to the obtained autopilots at different design trials which yields the reduced order controller as:

$$
C 1(\mathrm{red})=\frac{-122.1 s^{3}-8941 s^{2}-3.776 \mathrm{e}^{4} s-6887}{s^{3}+3.447 s^{2}+2.944 s+0.1019} .
$$




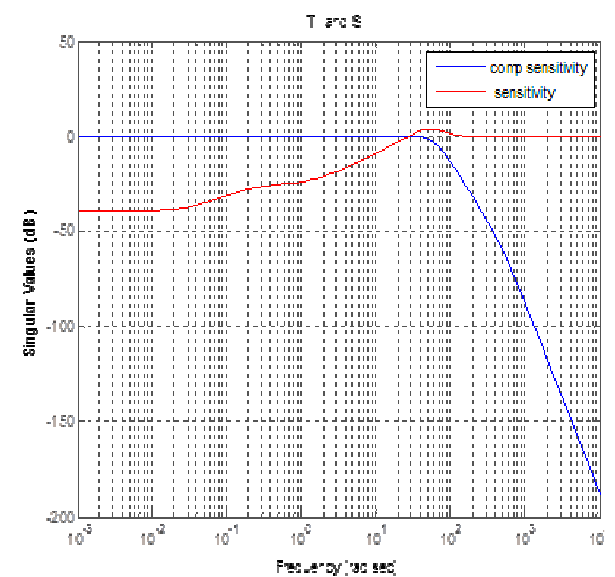

Fig. 3 a Sensitivity and Complementary weighting functions

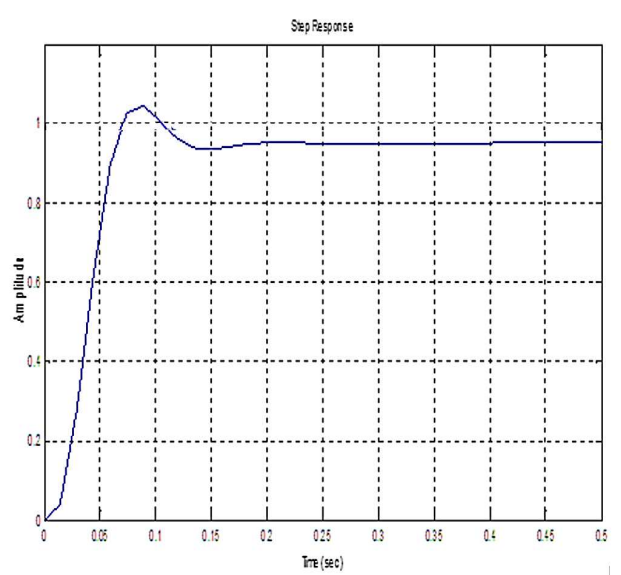

Fig. $3 b$ Step response of robust $C L S$

\section{b. Second Robust Controller}

A robust controller is designed using the following weights:

$$
W_{p}=\frac{0.59 s+20}{s+0.15}, W_{t}=\frac{0.03 s+1.2}{10 s+2}, W_{u}=\frac{0.001}{s+0.17} .
$$

The obtained $H_{\infty}$ controller has the form:

$$
C 2=\frac{-1.669 \mathrm{e}^{6} s^{5}-4.866 \mathrm{e}^{8} s^{4}-2.878 \mathrm{e}^{10} s^{3}-1.225 \mathrm{e}^{11} s^{2}-3.476 \mathrm{e}^{10} s-2.279 \mathrm{e}^{9}}{s^{6}+2345 s^{5}+2.724 \mathrm{e}^{6} s^{4}+6.688 \mathrm{e}^{6} s^{3}+3.435 \mathrm{e}^{6} s^{2}+4.237 \mathrm{e}^{5} s+4040} \text {. }
$$

The frequency response of the obtained weighted sensitivities is shown in Fig. 4a, and the closed loop response is shown in Fig. 4b. The reduced controllers are obtained as;

$$
C 2(\mathrm{red})=\frac{-937 s^{3}-3706 s^{2}-1.047 \mathrm{e} 005 s-1.107 \mathrm{e} 004}{s^{3}+5.483 s^{2}+1.944 s+0.01962} .
$$

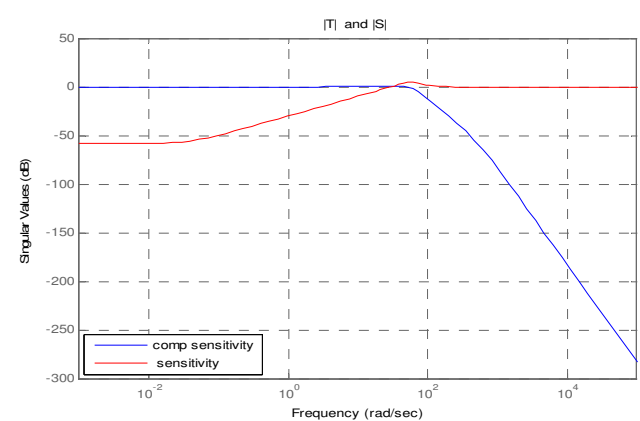

Fig. 4a Sensitivity and Complementary weighting functions

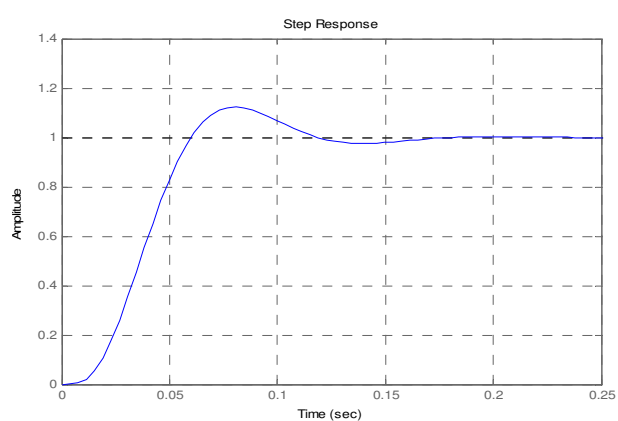

Fig. $4 b$ Step response of robust $C L S$ 


\subsection{Robust Controller Performance Evaluation}

For our underlying missile, the transfer function of the fin servo drive (control surface actuator) is given by:

$$
\frac{\delta}{\delta_{\text {ref }}}=\frac{0.175}{0.0133 s+1} .
$$

The extracted airframe transfer function has the form

$$
\frac{\theta(s)}{\delta_{p}(s)}=\frac{a_{\delta} s+a_{\delta} n_{\alpha}}{s^{3}+\left(n_{\alpha}-a_{\omega}\right) s^{2}-\left(a_{\omega} n_{\alpha}+a_{\alpha}\right) s},
$$

where the coefficients $a_{\alpha}, a_{\delta}, a_{\omega}, n_{\alpha}$ are obtained from the flight path parameters. The frequency response of the extracted airframe transfer function $\left(\theta / \delta_{p}\right)$ at different operating conditions is shown in Fig. 5. This figure clarifies that the max gain variation is $3 \mathrm{~dB}$ at low frequency and $28 \mathrm{~dB}$ at high frequency. In addition, the maximum phase variation is $2.2^{\circ}$ at low frequency and $64^{\circ}$ at high frequency. Moreover, the point number 6 is chosen based on that it has a moderate frequency response w.r.t other than obtained frequency response from obtained set points.

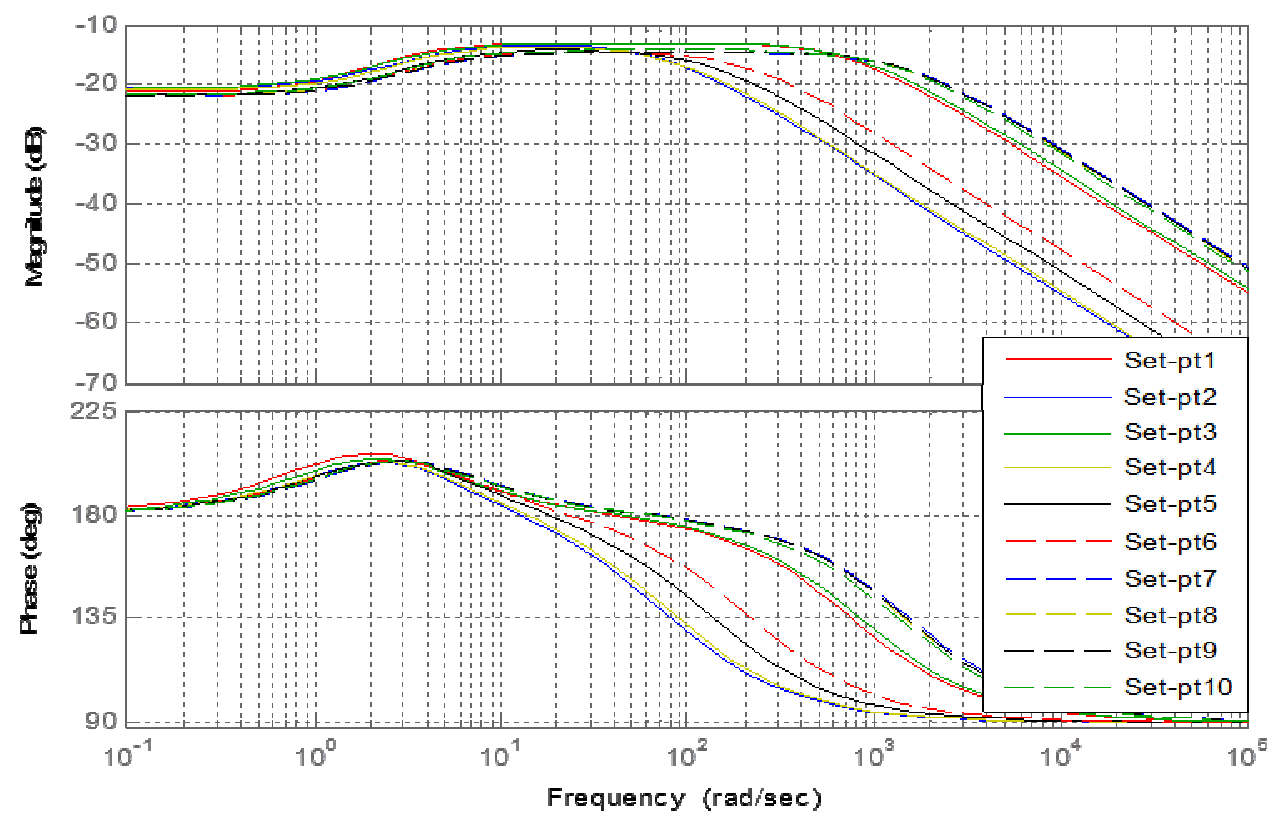

Fig. 5 Bode Diagram of the airframe $\left(\theta / \delta_{p}\right)$

Also, the unmolded dynamic is conceded by choosing the point number 3 and 9 which have different TF from the one used to design the controller.

\section{c. Non-modelled Dynamics}

The designed controllers are implemented with different operating points (including $3^{\text {rd }}$ and $9^{\text {th }}$ operating points). The obtained results are shown in Fig. 6a, b and Fig. 7a, b which clarify that the controller obtained taking the $6^{\text {th }}$ operating point as a nominal transfer function is not stable against all non-modelled dynamics when using the original autopilot while it is stable against all non-modelled dynamics for the designed robust autopilot. 


\section{For $3^{\text {rd }}$ Operating Point}

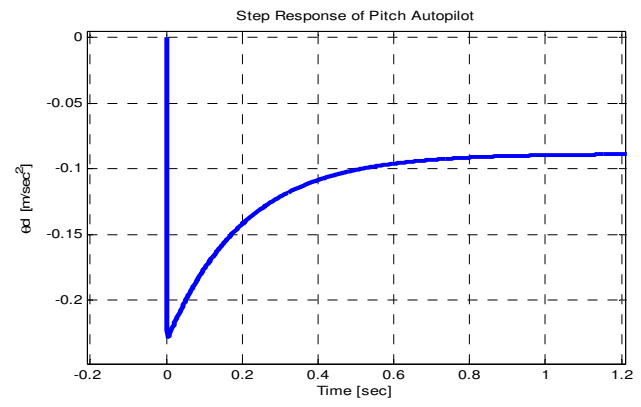

Fig. 6a Step response of the original autopilot

\section{For $9^{\text {th }}$ Operating Point}

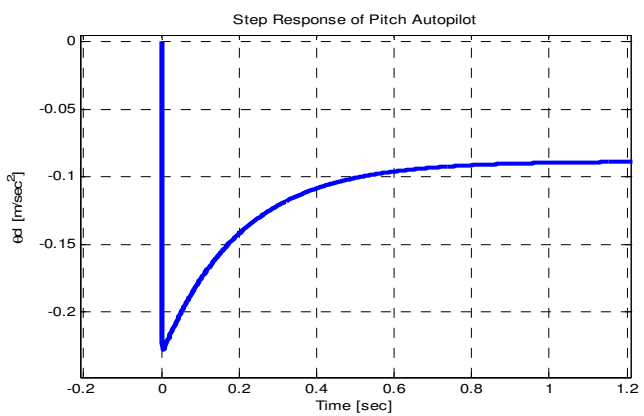

Fig. 7a Step response of the original autopilot

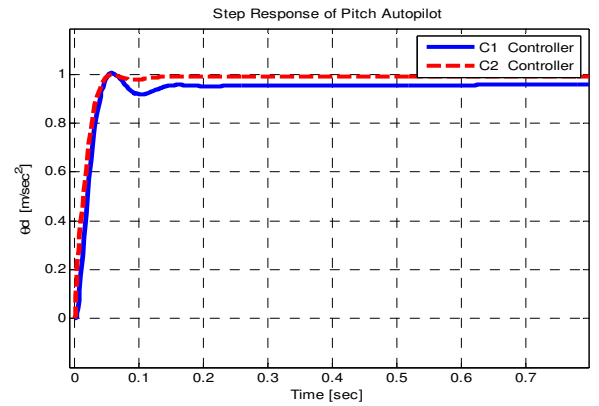

Fig. 6b Step response of the designed autopilot

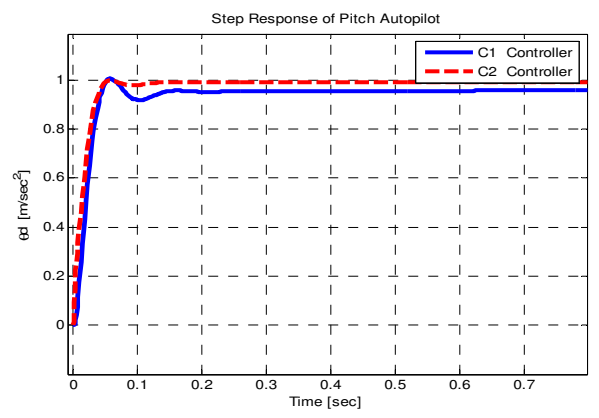

Fig. $7 b$ Step response of the designed autopilot

\section{d. Control Signal}

During the implementation of designed robust controllers, the obtained control signals are shown in Fig. 8. This figure clarifies that the designed controller $C 1$ is smoother than $C 2$, but with higher control signal at steady state.

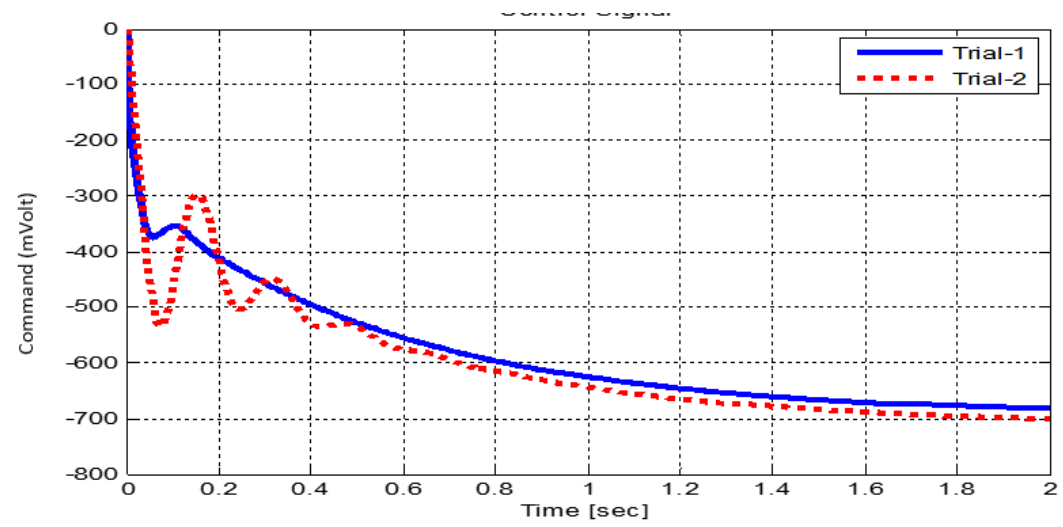

Fig. 8 Control signal using robust control 


\section{e. Disturbance Rejection}

Applying an impulse disturbance to the fin servo output yields the control efforts shown in Fig. 9, which clarifies that the convergence using $C 1$ after applying a disturbance is the best compared to the other controller.

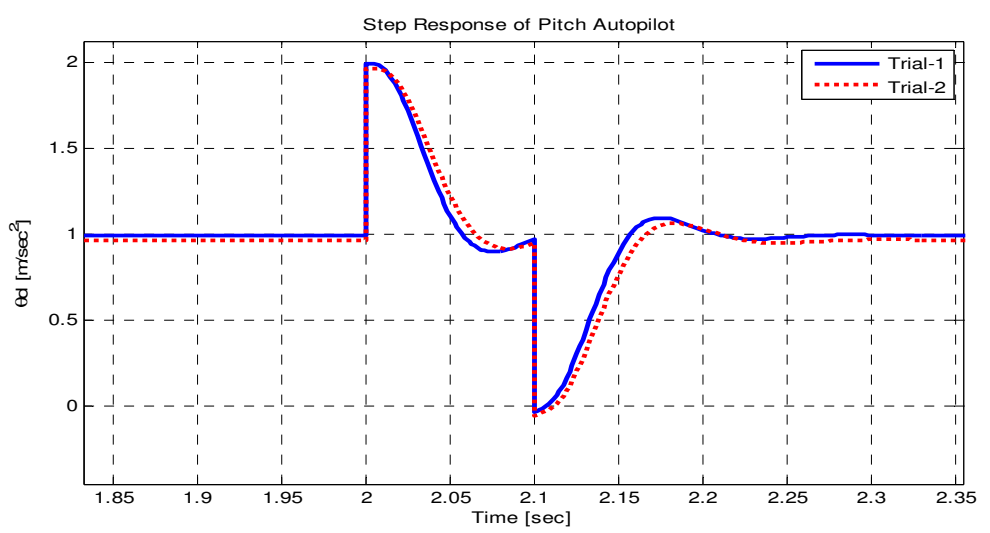

\section{f. Noise Sensitivity}

Fig. 9 Disturbance response using robust controllers

Applying a white Gaussian noise to the tracker output yields the control effort shown in Figure 10, which clarifies that $C 1$ has lower infinity norm $\left\|\omega_{t} T\right\|_{\infty}=0.2378$ than $C 2$ $\left\|\omega_{t} T\right\|_{\infty}=0.5623$ which has an indication of the stability of the robust controller and it is less sensitive to additive noise compared to other controllers.

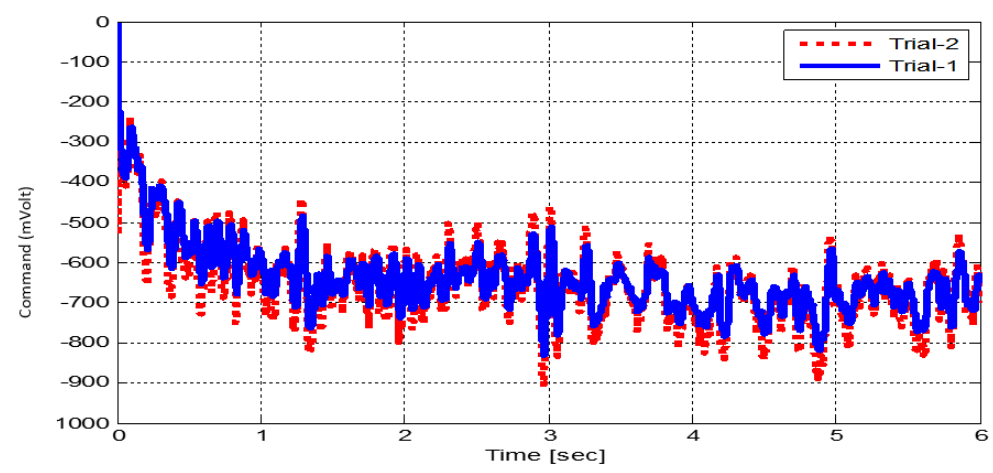

Fig. 10 Control signal using robust control

The above-mentioned figures reveal that the designed robust Trial-1 has an overshoot with the step response but it is less sensitive to the applied disturbance and noise and has a successful flight path trajectory compared to the second trial.

\section{Flight Path Evaluation}

\subsection{1 Robust Flight}

The designed robust controllers are converted to differential equations inside the $6 \mathrm{DOF}$ model, the simulation of which yields the flight path trajectories shown in Figs. 11-14. 


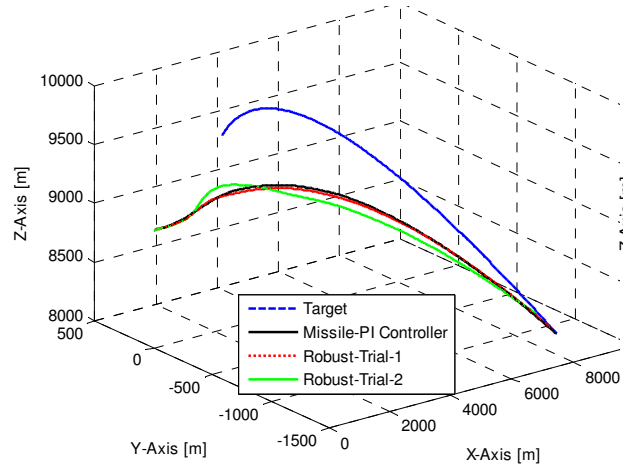

Fig. 11 Missile-target engagement scenario No. 1

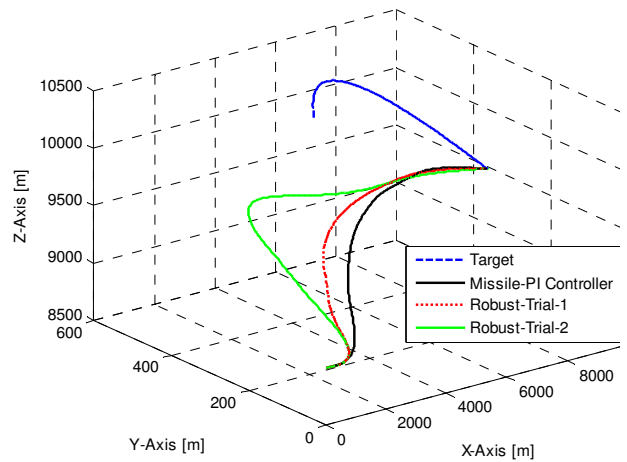

Fig. 13 Missile-target engagement scenario No. 3

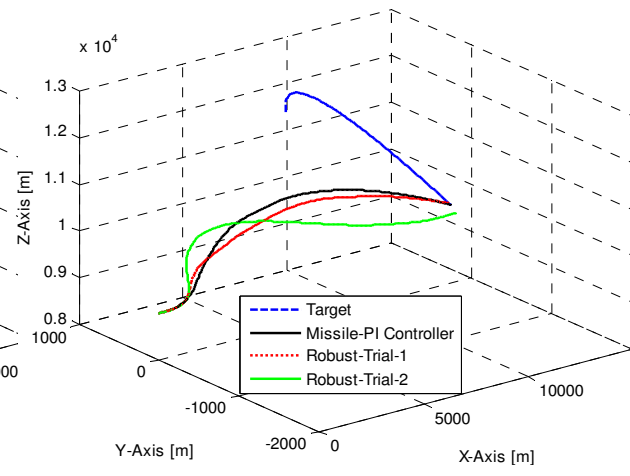

Fig. 12 Missile-target engagement scenario No. 2

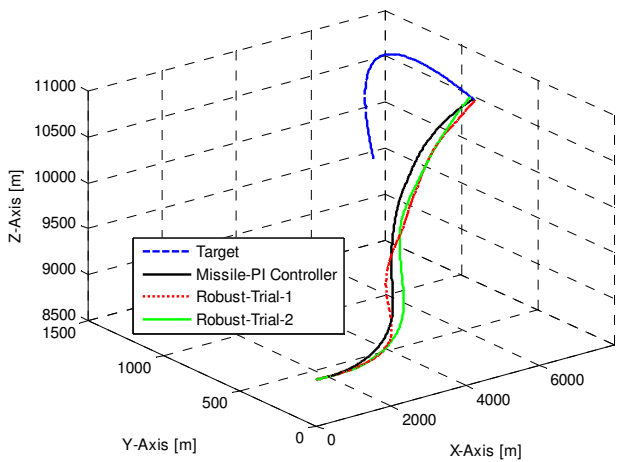

Fig. 14 Missile-target engagement scenario No. 4

From the engagement scenarios above, Tab. 1 shows the miss-distance which explains that the designed autopilot using robust controller has accepted miss-distance compared with the proportional controller.

Tab. 1 Miss-distance

\begin{tabular}{|c|c|c|}
\hline \multirow{2}{*}{ Engagement } & \multicolumn{2}{|c|}{ Miss-distance $[\mathrm{m}]$} \\
\cline { 2 - 3 } & $\begin{array}{c}\text { Proportional } \\
\text { controller }\end{array}$ & Robust controller \\
\hline Scenario-1 & 464.87 & 9.97 \\
\hline Scenario-2 & 1090.06 & 7.75 \\
\hline Scenario-3 & 20.70 & 9.28 \\
\hline Scenario-4 & 30.69 & 7.88 \\
\hline
\end{tabular}




\subsection{Robust and Classical Flight Comparison}

The flight paths obtained with both the classical and robust controllers / autopilots are shown in Fig. 15 which clarifies less demanded manoeuvre using the robust autopilot. In addition, Tab. 2 shows the comparison between the classical controller and the robust controller in view of flight variables.

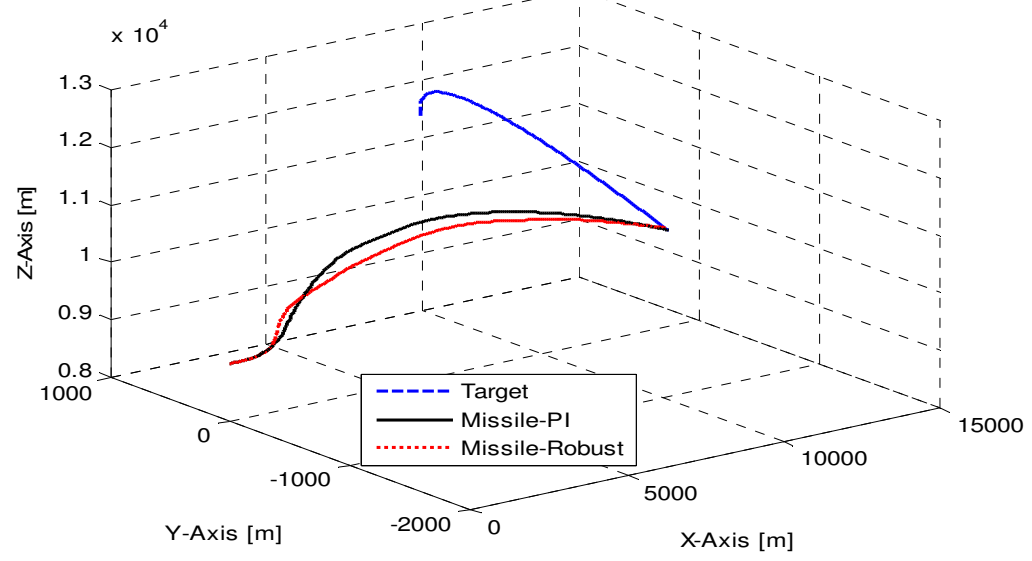

Fig. 15 Flight path using the classical and robust autopilots

Tab. 2 Controller comparison

\begin{tabular}{|l|r|r|}
\hline \multicolumn{1}{|c|}{ Performance } & Classical & Robust \\
\hline Miss distance & 8.820 & 9.970 \\
\hline Variance of control effort & 33700.000 & 5546.600 \\
\hline Variance of fin deflection & 12.750 & 1.880 \\
\hline Variance of normal acceleration & 0.084 & 0.004 \\
\hline Max normal acceleration $\left[\mathrm{m} / \mathrm{s}^{2}\right]$ & 163.630 & 165.510 \\
\hline Time of flight $[\mathrm{s}]$ & 15.170 & 15.060 \\
\hline
\end{tabular}

\section{Simulation with Hardware-in-the-Loop (HIL)}

The fin actuator simulation part is replaced by acquiring the hardware control section via a data acquisition card with the flight simulation model [17] as shown in Fig. 16. The results for the HIL experiments and the pure simulation for control section interior signals waveforms are discussed in next subsections.

\subsection{Applying PI Controller with HIL}

\section{a. HIL Evaluation in Noise Free Environment}

The difference between simulation and HIL results using PI controller is shown in Fig. 17, which represents the fin deflection with time. The evaluation is carried out by using HIL using different engagement scenarios, some of which are tabulated in Tab. 3. 


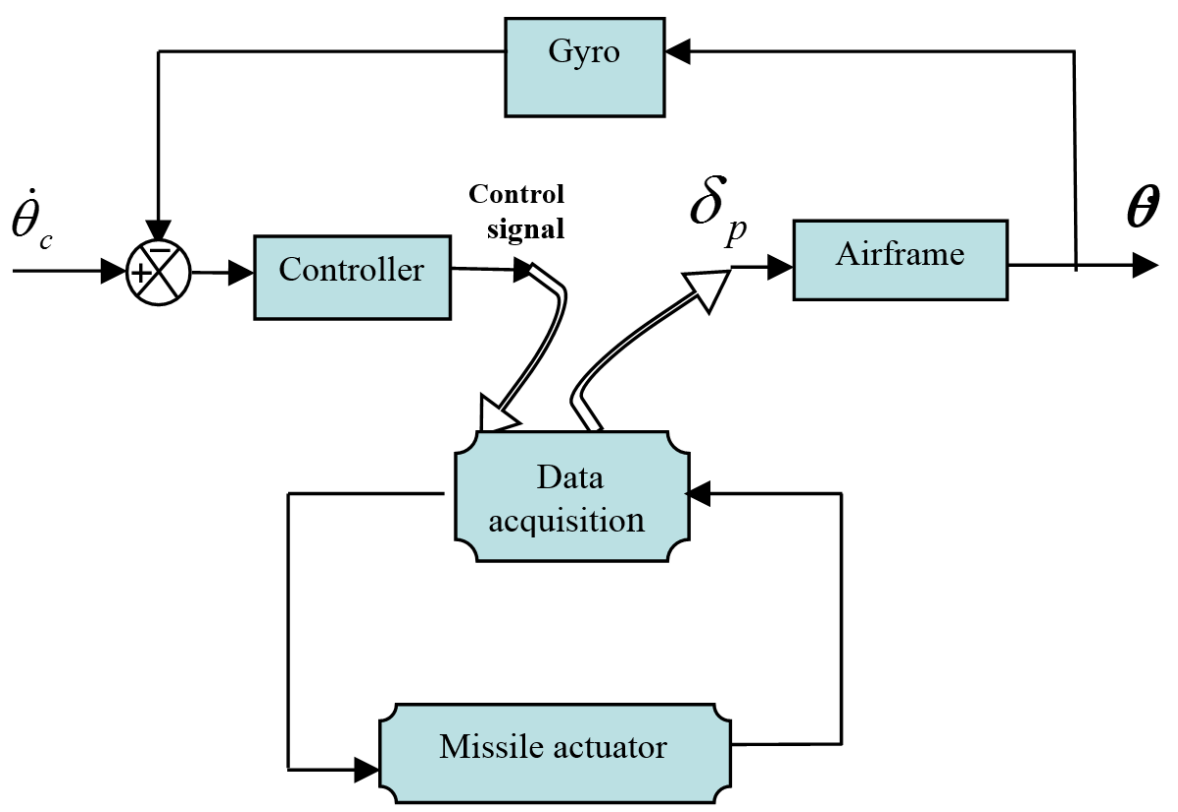

Fig. 16 Hardware in the loop

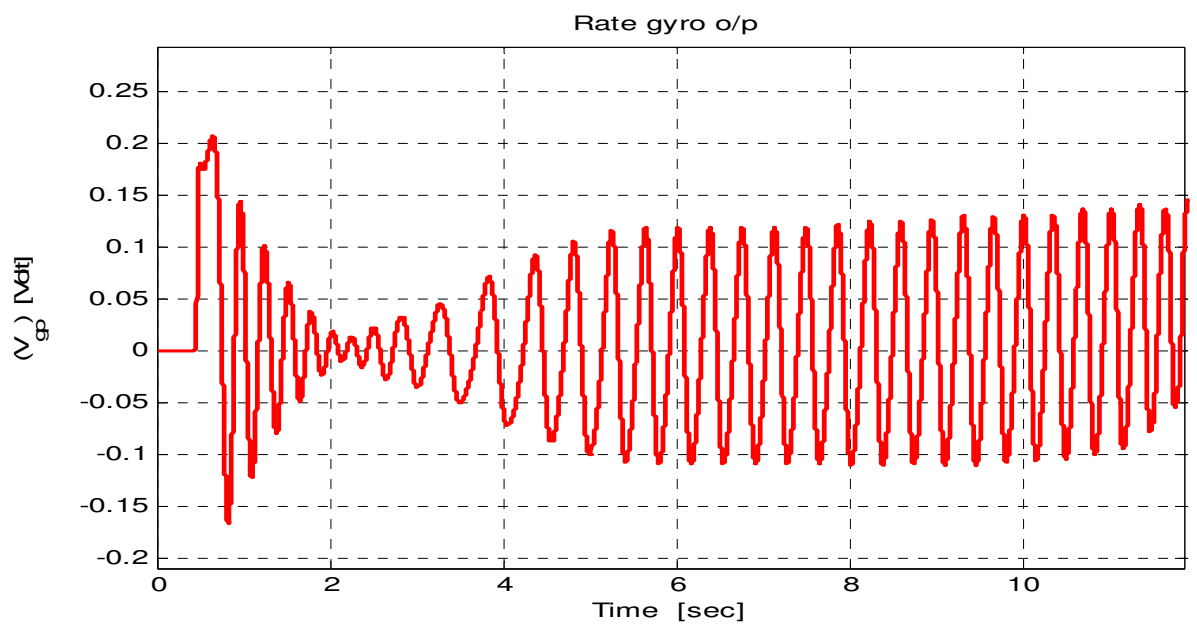

Fig. 17 Rate gyro output noise free

\section{b. HIL Evaluation in Presence of Noise}

The effect of adding noise to simulation model is shown in Fig. 18, which represents the effect of noise on the fin deflection signal that becomes noisy. The evaluation of the effect of this noise on the time of flight, miss distance and the normal acceleration is carried out using the HIL with different engagement scenarios as tabulated in Tab. 4. 
Tab. 3 Engagement scenarios for PI controller (noise free)

\begin{tabular}{|c|c|c|c|}
\hline Scenario & Miss-distance $[\boldsymbol{m}]$ & $\mathbf{T}_{\mathbf{f}}[\boldsymbol{s}]$ & $\mathbf{J}_{\mathbf{N}}[\boldsymbol{g}]$ \\
\hline 1 & 9.79 & 12.50 & 16.80 \\
\hline 2 & 9.64 & 15.16 & 17.83 \\
\hline 3 & 9.52 & 11.10 & 11.53 \\
\hline 4 & 9.50 & 11.10 & 15.36 \\
\hline
\end{tabular}

Tab. 4 Engagement scenarios for using PI controller (noisy)

\begin{tabular}{|c|c|c|c|}
\hline Scenario & Miss-distance $[\boldsymbol{m}]$ & $\mathbf{T}_{\mathbf{f}}[\boldsymbol{s}]$ & $\mathbf{J}_{\mathbf{N}}[\boldsymbol{g}]$ \\
\hline 1 & 9.99 & 12.49 & 10.64 \\
\hline 2 & 9.79 & 15.15 & 17.53 \\
\hline 3 & 9.70 & 11.10 & 14.90 \\
\hline 4 & 9.89 & 10.71 & 15.54 \\
\hline
\end{tabular}

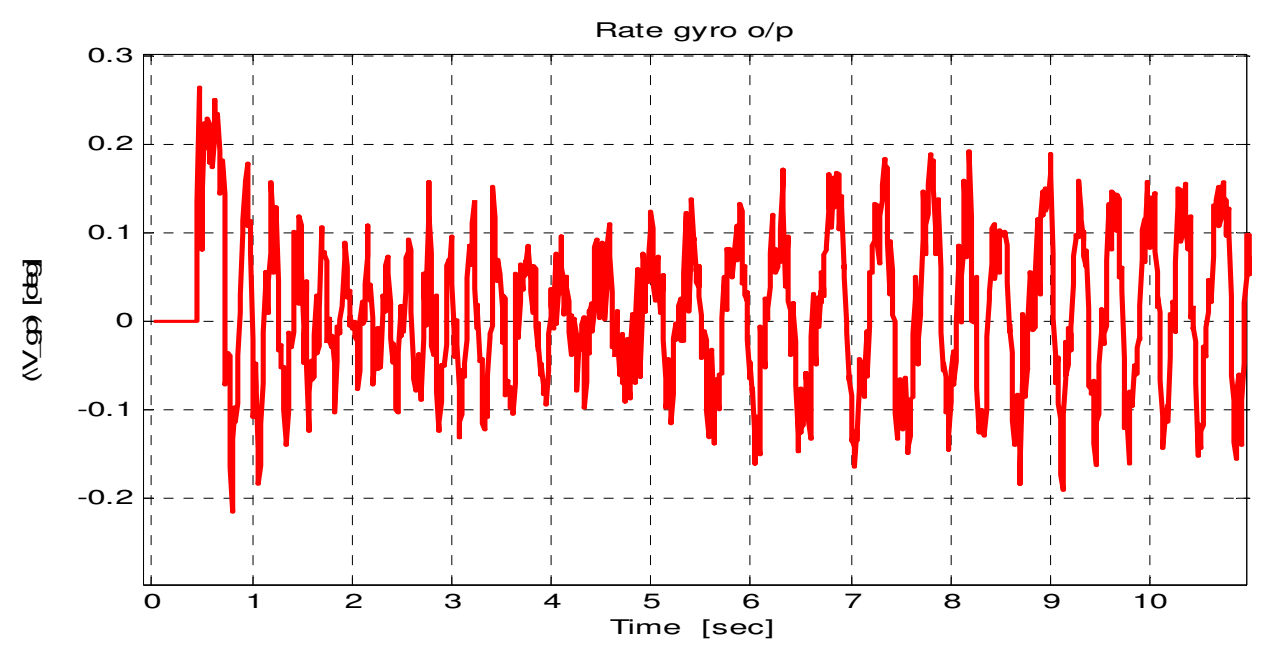

Fig. 18 Rate gyro output noisy

\section{c. HIL Evaluation in Presence of Disturbance}

The fin deflection signal after applying a disturbance is shown in Fig. 19, where Fig. 19a shows the fin deflection signal with no feedback from $(3 \div 3.8 \mathrm{~s})$ and $(6.6 \div 7.8 \mathrm{~s})$, while Fig. $19 \mathrm{~b}$ represents the disturbance effect that applied from $(3.8 \div 5.4 \mathrm{~s})$. The evaluation of the disturbance effect on the flight time, miss distance and the normal acceleration is carried out using the HIL with different engagement scenarios as tabulated in Tab. 5 . 


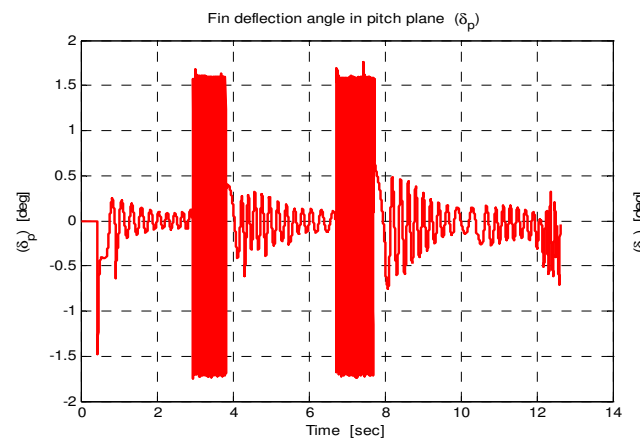

Fig. 19a Fin deflection with disturbance HIL

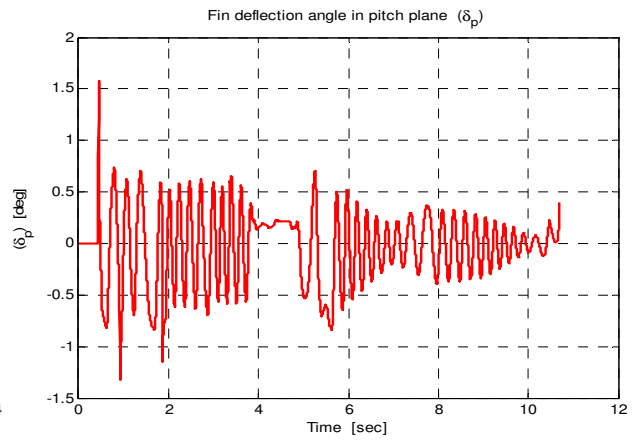

Fig. 19b Fin deflection with disturbance HIL

Tab. 5 Engagement scenarios using PI controller (with disturbance)

\begin{tabular}{|c|c|c|c|}
\hline Scenario & Miss-distance $[\boldsymbol{m}]$ & $\mathbf{T}_{\mathbf{f}}[\boldsymbol{s}]$ & $\mathbf{J}_{\mathbf{N}}[\boldsymbol{g}]$ \\
\hline 1 & 9.99 & 12.51 & 15.78 \\
\hline 2 & 9.93 & 11.11 & 15.41 \\
\hline 3 & 9.47 & 11.09 & 13.01 \\
\hline 4 & 9.87 & 11.09 & 13.12 \\
\hline
\end{tabular}

\section{d. Evaluation with Flight Path}

Fig. 20 above reveals that the designed PI controller used within the HIL has different characteristics in the presence of disturbance and noise compared to pure simulation. Also, the controller has a successful flight path trajectory except the designed PI-2 has an unstable trajectory and highest miss-distance.

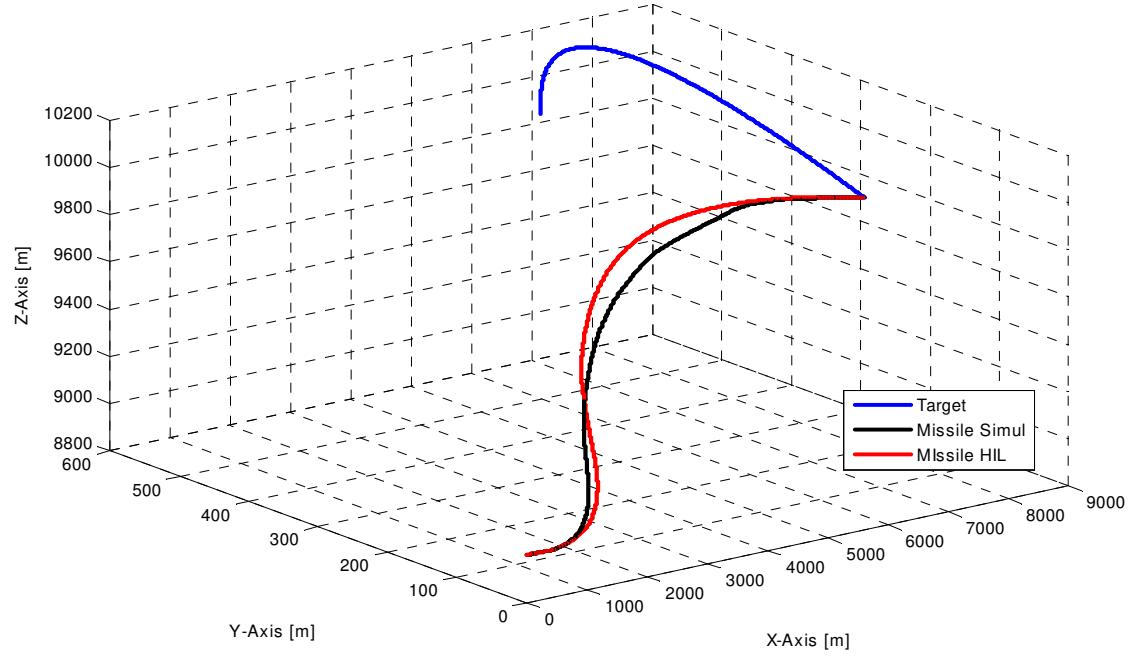

Fig. 20 Missile-target engagement scenario with PI and HIL 


\subsection{Applying Robust Controller with HIL}

\section{a. HIL Evaluation in Noise Free Environment}

The difference between simulation and HIL results using robust controller is shown in Fig. 21, which represents the fin deflection signal with time. The evaluation is carried out by using HIL with different engagement scenarios, some of which are tabulated in Tab. 6.

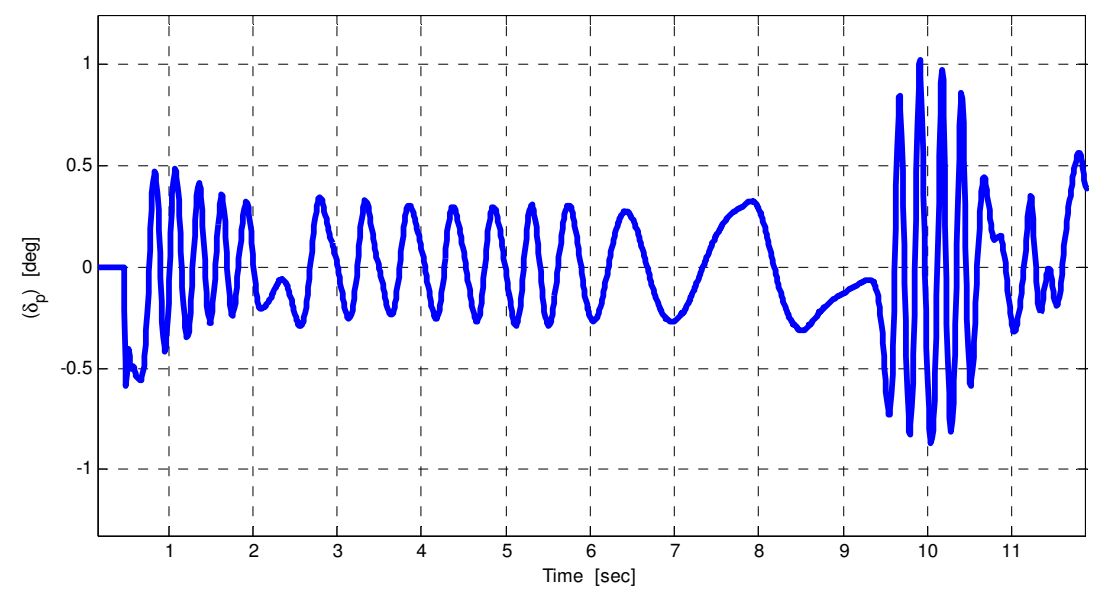

Fig. 21 Rate gyro output (noise free)

Tab. 6 Engagement scenarios using robust controller (noise free)

\begin{tabular}{|c|c|c|c|}
\hline Scenario & Miss-distance $[\boldsymbol{m}]$ & $\mathbf{T}_{\mathbf{f}}[\boldsymbol{s}]$ & $\mathbf{J}_{\mathbf{N}}[\boldsymbol{g}]$ \\
\hline 1 & 9.994 & 12.570 & 17.971 \\
\hline 2 & 9.984 & 15.062 & 11.445 \\
\hline 3 & 9.999 & 11.148 & 15.938 \\
\hline 4 & 9.973 & 10.676 & 17.736 \\
\hline
\end{tabular}

\section{b. HIL Evaluation in Presence of Noise}

The noise effect on the simulation model is shown in Fig. 22 which represents the effect of noise on the fin deflection signal that becomes noisy. The evaluation of the effects of this noise is tabulated in Tab. 7.

Tab.7 Engagement scenarios using Robust controller (noisy)

\begin{tabular}{|c|c|c|c|}
\hline Scenario & Miss-distance $[\boldsymbol{m}]$ & $\mathbf{T}_{\mathbf{f}}[\boldsymbol{s}]$ & $\mathbf{J}_{\mathbf{N}}[\boldsymbol{g}]$ \\
\hline 1 & 9.76 & 12.51 & 14.06 \\
\hline 2 & 8.87 & 15.13 & 10.81 \\
\hline 3 & 7.87 & 11.13 & 13.63 \\
\hline 4 & 9.03 & 10.73 & 14.01 \\
\hline
\end{tabular}




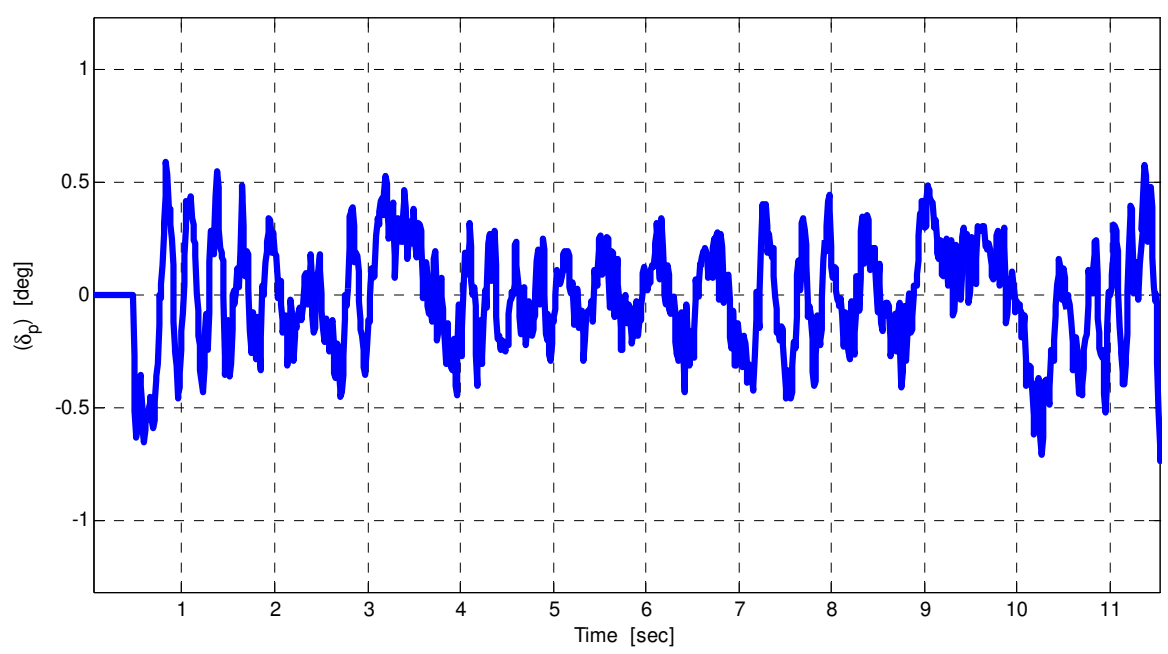

Fig. 22 Rate gyro output noisy

\section{c. HIL Evaluation In Presence of Disturbance}

Fig. 23 shows the fin deflection signal with the effect of applying disturbance. The evaluation is carried out using different engagement scenarios, some of which are tabulated in Tab. 8 .

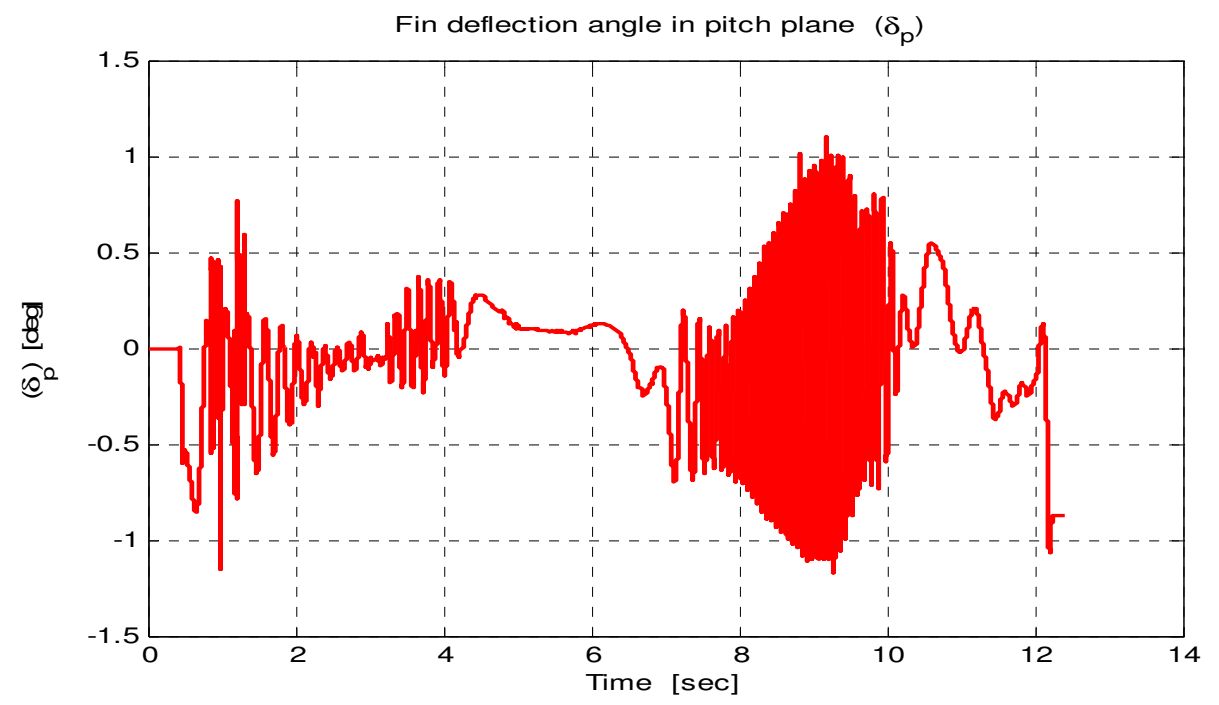

Fig. 23 Fin deflection with disturbance

\section{d. Evaluation with the Flight Path Visualization}

Fig. 24 reveals that the designed robust controller using the HIL has different characteristics in the presence of disturbance and noise compared to pure simulation. Also, the controller has a successful flight path trajectory except the designed robust Trial-2 which has an unstable trajectory and highest miss-distance. 
Tab. 8 Engagement scenarios using Robust (with disturbance)

\begin{tabular}{|c|c|c|c|}
\hline Scenario & Miss-distance $[\boldsymbol{m}]$ & $\mathbf{T}_{\mathbf{f}}[\boldsymbol{s}]$ & $\mathbf{J}_{\mathbf{N}}[\boldsymbol{g}]$ \\
\hline $\mathbf{1}$ & 9.98 & 15.06 & 11.44 \\
\hline $\mathbf{2}$ & 9.99 & 12.57 & 17.97 \\
\hline $\mathbf{3}$ & 9.99 & 11.15 & 15.94 \\
\hline $\mathbf{4}$ & 9.97 & 10.68 & 17.74 \\
\hline
\end{tabular}

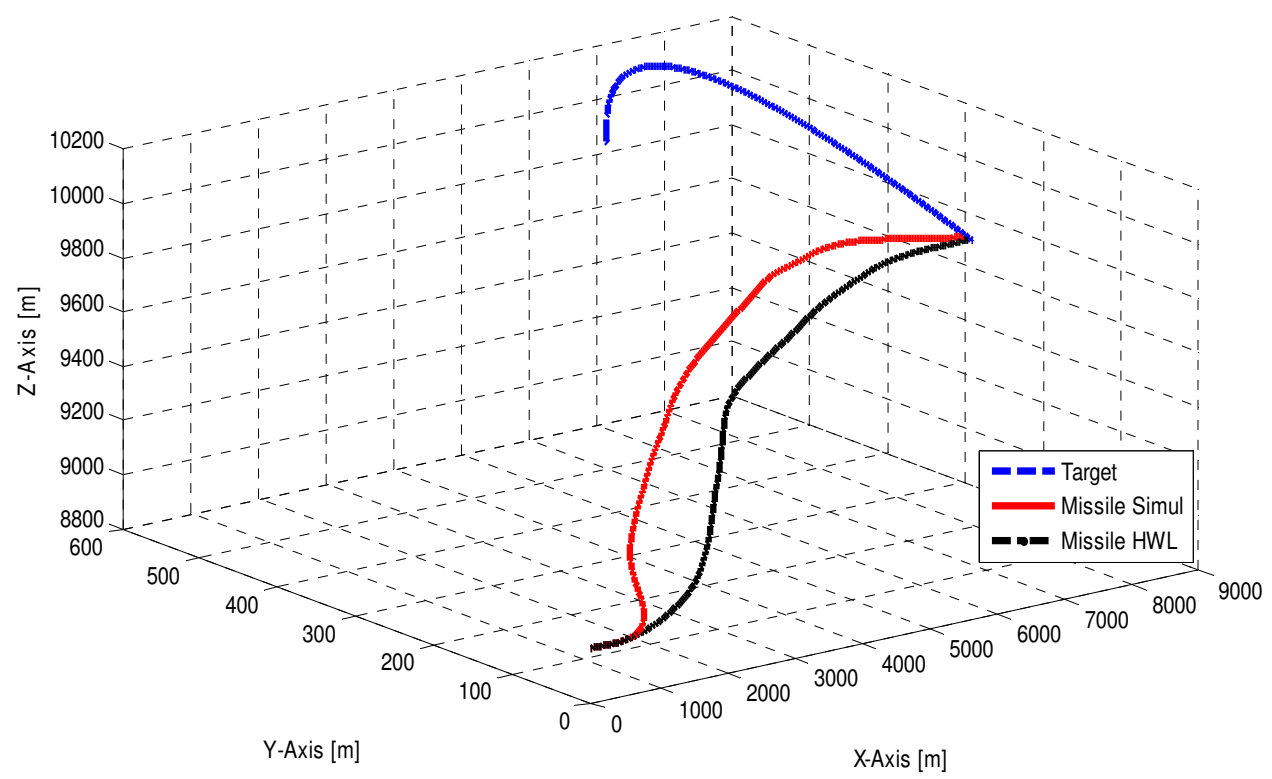

Fig. 24 Missile-target engagement scenario

\section{Conclusion}

This work has the advance in developing a Sidewinder missile from the first generation. In addition, the main advantage of this work is the application itself which was conducted with a real missile still in the service. The flight path evaluation considering the software environment reveals that the designed robust autopilot has a successful flight path trajectory against different types of disturbance and noise, as well as it has acceptable miss-distance. Furthermore, the control effort obtained using robust controller is lower than that obtained with proportional controller. To achieve good and enhanced performance, the system modelling should be carried out via the HILS. The PI and robust controller are evaluated against the noise and disturbance within the HIL environment. The flight path evaluation has shown that the designed robust autopilot has a successful flight path trajectory in presence of disturbance and noise, with acceptable miss-distance and lower control effort than that obtained with classical controller by $25 \%$. 


\section{Acknowledgement}

The authors wish to express their gratitude to the Egyptian Armed Forces for the financial support extended to this research project.

\section{References}

[1] AZAR, A.T. and ZHU, Q. Advances and Applications in Sliding Mode Control Systems. Berlin: Springer, 2015. DOI 10.1007/978-3-319-11173-5.

[2] GABREL, V., MURAT, C. and THIELE, A. Recent Advances in Robust Optimization: An overview. European Journal of Operational Research, 2014, vol. 235, no. 3, p. 471-483.

[3] RASTEgAR, S., ARAÚJO, R., EMAM, I.A. and IRATNI, A. A New Robust Control Scheme for LTV Systems Using Output Integral Discrete Synergetic Control Theory. In: Garrido, P., Soares, F. and Moreira, A. (eds) CONTROLO 2016. Lecture Notes in Electrical Engineering, vol. 402, Cham: Springer, 2017, p. 117-127. DOI 10.1007/978-3-319-43671-5_11.

[4] LI, S. and YANG, J. Robust Autopilot Design for Bank-to-turn Missiles using Disturbance Observers. In IEEE Transactions on Aerospace and Electronic Systems, 2013, vol. 49, no. 1, p. 558-579. DOI 10.1109/TAES.2013.6404120.

[5] MATTEI, G. and MONACO, S. Nonlinear Autopilot Design for an Asymmetric Missile using Robust Backstepping Control. Journal of Guidance, Control and Dynamics, 2014, vol. 37, no. 5, p. 1462-1476. DOI 10.2514/1.G000434.

[6] LIU, X., LIU, Z., SHAN, J. and Sun, H. Anti-disturbance Autopilot Design for Missile System via Finite Time Integral Sliding Mode Control Method and Nonlinear Disturbance Observer Technique. Transactions of the Institute of Measurement and Control, 2016,vol. 38, no. 6, p. 693-700. DOI 10.1177/0142331215603793.

[7] LEE, K.W. and SINGH, S.N. Longitudinal Nonlinear Adaptive Autopilot Design for Missiles with Control Constraint. Proceedings of the Institution of Mechanical Engineers, Part G: Journal of Aerospace Engineering, 2017. DOI 10.1177/0954410017699002.

[8] MOHAMED, A., EL-SHEIKH, G.A., YOUSEF, A.M. and OUDA, A.N. Classical Autopilot Design for Air to Air Guided Missile: Flight Path Synthesis. International Journal of Engineering System Modelling and Simulation. 2017.

[9] OUDA, A.N. Performance Investigation of Adaptive Guidance Algorithms and its Effectiveness [PhD Thesis]. Cairo: Military Technical College, 2012.

[10] Math Works, Inc., Robust Control Toolbox. User's Guide. 1992 - 2001.

[11] SIOURIS, G.M. Missile Guidance and Control Systems. New York: Springer, 2004, 666 p., ISBN 978-0-387-00726-7.

[12] DOLYE, J., FRANCIS, B. and TANNENBAUM, A. Feedback Control Theory. MacMillan, 1990.

[13] SAFONOV, M.G. and CHIANG, R.Y. A Schur Method for Balanced Model Reduction. IEEE Transactions on Automatic Control, 1989, vol. 34, no. 7, p. 729-733. DOI 10.1109/9.29399. 
[14] LIM, K.B. and GAWRONSKI, W. Hankel singular values of flexible structures in discrete time. In Guidance, Navigation, and Control Conference, Guidance, Navigation, and Control and Co-located Conferences, p. 1370-1377, 1996. DOI 10.2514/6.1996-3757.

[15] MOHAMED A. Automatic Control of Guided Missile [MSc Thesis]. Cairo: Military Technical College, 2012.

[16] HALIKAS, G.D. An Affine Parametrization of all One-block $\mathrm{H}_{\infty}$ Optimal Matrix Interpolating Functions. International Journal of Control. 1993, vol. 57, no. 6, p. 1421-1441. DOI 10.1080/00207179308934455.

[17] SUTTON, R.P., HALIKIAS, G.D., PLUMMER, A.R. and WILSON, D.A. Robust Control of Lightweight Flexible Manipulator under the Influence of Gravity. In IEEE International Conference on Control Application, 1997, p. 300-305. DOI 10.1109/CCA.1997.627559. 